\title{
Atomic limit and inversion-symmetry indicators for topological superconductors
}

\author{
Anastasiia Skurativska $\odot$, Titus Neupert, and Mark H. Fischer \\ Department of Physics, University of Zurich, Winterthurerstrasse 190, 8057 Zurich, Switzerland
}

(Received 1 July 2019; revised manuscript received 25 November 2019; published 21 January 2020)

\begin{abstract}
Symmetry indicators have proven to be extremely helpful in identifying topological crystalline insulators using symmetry-group representations of their Bloch states. An extension of this approach to superconducting systems requires defining an appropriate atomic limit for Bogoliubov-de-Gennes Hamiltonians. Here, we introduce such a notion of atomic limit and derive a $\mathbb{Z}_{2^{d}}$-valued symmetry indicator for inversion-symmetric superconductors in $d$ dimensions. This indicator allows for a refined topological classification including higher-order phases for systems in the superconducting symmetry classes D and DIII. We further elucidate their bulk-boundary correspondence using Dirac surface theories. Requiring only the normal-state band structure and the superconducting order-parameter symmetry as input, this indicator is well suited for a search of topological superconductors using first-principles calculations.
\end{abstract}

DOI: 10.1103/PhysRevResearch.2.013064

\section{INTRODUCTION}

Topological superconductors (TSCs) are of fundamental interest due to Majorana anyon modes on their boundaries [1]. These topologically protected modes cannot be removed from the boundary without breaking their protecting symmetries or closing the gap [1-3]. Unlike topological insulators, only few candidate TSCs exist [4].

Majorana modes are the only anyons admitting a description in terms of free fermions. Thus, a description of TSCs in terms of quadratic Bogoliubov-de-Gennes (BdG) Hamiltonians suffices for most purposes. By construction, these Hamiltonians posses particle-hole symmetry (PHS). Here, we are interested in topological phases of generic spin-orbit coupled TSCs with or without time-reversal symmetry (TRS), corresponding to symmetry classes D and DIII in the Altland Zirnbauer (AZ) scheme [5,6], respectively. Both classes support topological superconducting phases in one dimension (1D) and two dimensions (2D), while in three dimensions (3D), only class DIII allows for such a phase.

Two recent developments motivate our study: First, including crystalline symmetries the bulk-boundary correspondence for topological phases is very rich, including corner states in $2 \mathrm{D}$ and hinge or corner states in $3 \mathrm{D}$, instead of edge and surface states, respectively. Systems with such a generalized bulk-boundary correspondence are termed higher-order topological insulators/superconductors [7-18]. Second, several advances simplify the determination of topological phases from bulk electronic properties using group-representation approaches [12,19-22]. In particular, symmetry indicators based on early work of Fu and Kane [23] distinguish topological

Published by the American Physical Society under the terms of the Creative Commons Attribution 4.0 International license. Further distribution of this work must maintain attribution to the author(s) and the published article's title, journal citation, and DOI. phases through the transformation properties of the occupied electronic states, rather than lengthy calculations of topological indices. These properties are easily accessible from firstprinciples calculations using density functional theory (DFT).

Symmetry indicators quantify a mismatch between the real space representation in terms of localized Wannier orbitals and the momentum representation of (Bloch) bands [12,20]. Importantly, the atomic insulator, in other words localized Wannier orbitals without mutual overlap, defines an atomic limit (AL) of trivial bands. A gapped phase is considered to be topologically nontrivial if it cannot be adiabatically connected to such an AL without closing the bulk gap. The proper definition of an AL for insulators promotes the inversion-symmetry indicator to a $\mathbb{Z}_{4}$ quantity in 3D [12], compared to the $\mathbb{Z}_{2}$ valued Fu-Kane indicator [23]. Group representation approaches have been successful in predicting topological crystalline insulators and higher-order TIs from material databases [24-26].

Adapting an analogous approach of symmetry indicators for TSCs applicable within a DFT approach, one faces several challenges. First, the band structure of a material in the superconducting phase is not available from DFT calculations. A symmetry indicator, thus, should be defined in terms of the symmetry representations of the normal-metal bands, rather than those of the BdG Hamiltonian, with the order-parameter symmetry as an input. Further, on a practical level, the electronic spectrum is generically not bounded from above, which means the particle-hole-doubled BdG band structure is not bounded from below. These problems have been addressed in previous work [27]. However, the superconducting spectrum of the BdG Hamiltonian is the excitation spectrum of the (particle-hole) Nambu space and not the electron excitation spectrum of an insulator with PHS. Therefore an (trivial) "atomic" limit for superconductors needs to be specified.

In this work, we show that the irreducible representations of the $\mathrm{BdG}$ band structure alone are insufficient to formulate a comprehensive symmetry indicator for topological superconductors. Instead, we introduce an AL for superconductors 
in terms of the normal metal bands and with respect to that we define the symmetry indicators for (higher-order) TSC in classes D and DIII. For concreteness, we focus in the following on the case of inversion symmetry. Unlike the symmetry indicators for TIs, these indicators are $\mathbb{Z}_{8}$ valued in $3 \mathrm{D}$. We show that the symmetry indicators are consistent with the existing topological invariants for the strong TSC phases and agree with the previous results regarding the Fermi surface topology [28-31]. We demonstrate the predicted higher-order topological bulk-boundary correspondence with surface Dirac Hamiltonians.

\section{MAIN RESULT}

Our main finding is that for odd-parity order parameters, the symmetry indicator

$$
\kappa_{d, \xi}=r_{\xi} \sum_{k \in \mathrm{TRIM}}\left(n_{k, \mathrm{~N}}^{+}-n_{k, \mathrm{~N}}^{-}\right) \in \mathbb{Z}
$$

is a $\mathbb{Z}_{2^{d}}$ valued strong topological invariant for $d$-dimensional weak-coupling superconductors protected by inversion symmetry in symmetry class $\xi \in\{\mathrm{D}, \mathrm{DIII}\} .{ }^{1}$ Here, $n_{k, \mathrm{~N}}^{ \pm}$is the number of occupied states with inversion eigenvalue \pm 1 at the time-reversal-invariant momentum (TRIM) $\boldsymbol{k}$ in the normalstate band structure. ${ }^{2}$ The prefactors $r_{\mathrm{D}}=1$ and $r_{\mathrm{DIII}}=1 / 2$ ensure that $\kappa_{d, \xi}$ is integer valued and $r_{\mathrm{DIII}}=r_{\mathrm{D}} / 2$ avoids double counting due to Kramer's degeneracy in the TRS case. Note that in 3D for a normal state with TRS (class AII), $\kappa_{3 \text {,DIII }}=2 \kappa_{1}$, with $\kappa_{1}$ defined as in Ref. [12]. Further, this indicator can be decomposed as

$$
\kappa_{d, \xi} \bmod 2^{d}=\sum_{n=1}^{d} v_{d, n, \xi} 2^{n-1},
$$

where $v_{d, n, \xi} \in\{0,1\}$ are $\mathbb{Z}_{2}$ indices for $n$ th-order topology in $d$ dimensions. For $v_{d, n, \xi}=1$, the "minimal" topology of the system is an $n$ th-order topological phase, as illustrated in Table $\mathrm{I}^{3}$ In particular, the indices $v_{d, 1, \xi}=1$ pertain to topological phases not requiring inversion for their protection. The indices $v_{2,2, \xi}$ and $v_{3,3, \xi}$ describe higher-order TSCs with corner Majorana modes and Kramer's pairs thereof. Finally, $v_{3,2, \xi}=1$ indicates a second-order TSC with 1D chiral (helical) Majorana edge modes in class D (DIII). Note that cases of $d=n$ are not captured by the indicators based on a pure BdG formulation introduced recently [27].

\section{ATOMIC LIMIT AND SYMMETRY INDICATORS}

A quadratic Hamiltonian describing a noninteracting insulator is written in terms of electronic wave functions and

\footnotetext{
${ }^{1}$ Note that Eq. (1) is of the same form as the result obtained in Ref. [27] derived for $3 \mathrm{D}$, but attains a refined interpretation due to our definition of atomic limit.

${ }^{2}$ These are the momenta whose little group contains inversion, namely $k \in\{0, \pi\}$ in $1 \mathrm{D}, \boldsymbol{k} \in\{(0,0),(0, \pi),(\pi, 0),(\pi, \pi)\}$ in $2 \mathrm{D}$, and $\boldsymbol{k} \in\{(0,0,0),(0,0, \pi),(0, \pi, 0),(\pi, 0,0),(0, \pi, \pi),(\pi, 0, \pi)$, $(\pi, \pi, 0),(\pi, \pi, \pi)\}$ in $3 \mathrm{D}$.

${ }^{3}$ Note the similarity to the subgroup structure introduced in Ref. [10].
}

TABLE I. Illustration of the minimal topology indicated by $v_{d, n, \xi}=1$ with surface $(n=1)$, hinge $(n=2)$, and corner Majorana modes $(n=d)$. In class DIII in $3 \mathrm{D}$ and class $\mathrm{D}$ in $2 \mathrm{D}$, which have $\mathbb{Z}$ topological invariants, the indicators can not preclude first-order phases. Class D in 3D does not allow for a topological invariant in the tenfold way, but $v_{3,1, D}=1$ indicates (bulk) Weyl nodes in a translationally symmetric system.

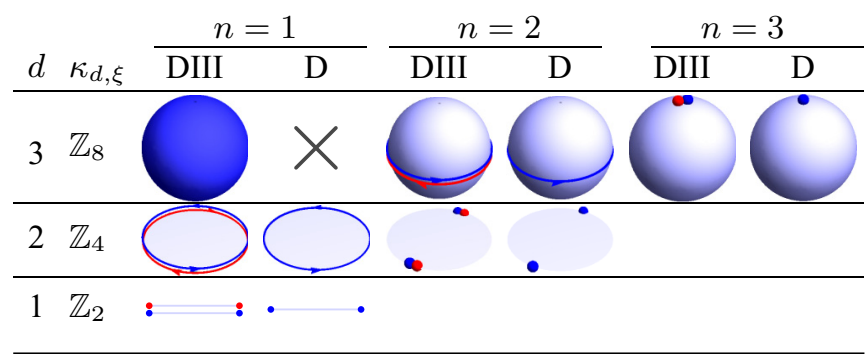

thus possesses a natural AL ground state, namely, the product state of independent occupied Wannier orbitals. In contrast, the ground state of a BdG Hamiltonian cannot be written as a direct product state with Bogoliubov quasiparticles as local physical degrees of freedom. This calls for an alternative definition of AL for superconductors. Our strategy to define such an AL is to find a BdG Hamiltonian, where $\Delta_{k} \rightarrow 0$ does not close a gap and whose normal state possesses an AL in the sense of Ref. [12]. We then ask whether we can connect this Hamiltonian, which we denote as the AL of the superconductor, adiabatically to the original BdG Hamiltonian.

For a quadratic Hamiltonian (with the chemical potential $\mu=\epsilon_{\mathrm{F}}$, the Fermi energy), the normal-state band structure is bounded from below and we can move $\mu$ below the lowest band of the normal state to find a topologically trivial normal state Hamiltonian (we write $\mu=-\infty$ ). The corresponding BdG Hamiltonian is then trivial in the above sense and serves in the following as our reference state, or AL. To quantify the mismatch between the two Hamiltonians, we introduce a symmetry indicator as the difference in the number of irreducible representations of the occupied BdG bands $\left.n_{k, \mathrm{BdG}}^{\alpha}\right|_{\mu=\epsilon_{\mathrm{F}}}$ (ana$\log$ to $\left.\kappa_{1}[12]\right)$ and of the occupied bands corresponding to the AL $\left.n_{k, \text { BdG }}^{\alpha}\right|_{\mu=-\infty}$. Specifically, the (inversion) symmetry indicator is defined as

$$
\kappa_{d, \xi}=r_{\xi} \sum_{k \in \text { TRIMs }} \sum_{\alpha \in\{ \pm 1\}} \frac{\alpha}{2}\left(\left.n_{k, \mathrm{BdG}}^{\alpha}\right|_{\mu=\epsilon_{\mathrm{F}}}-\left.n_{k, \mathrm{BdG}}^{\alpha}\right|_{\mu=-\infty}\right),
$$

where $\alpha$ refers to the inversion eigenvalue. For general point group elements, a short discussion of symmetry indicators is given in Appendix.

To calculate this difference, we need the irreducible BdG band representations. Given the normal-state Hamiltonian $H_{k}$ and the (mean-field) superconducting order parameter $\Delta_{k}$, the superconductor is described by the BdG Hamiltonian

$$
H_{k}^{\mathrm{BdG}}=\left(\begin{array}{cc}
H_{k} & \Delta_{k} \\
\Delta_{k}^{\dagger} & -H_{-k}^{*}
\end{array}\right)
$$

Under any element of the generating point group, $g \in \mathcal{G}$, this Hamiltonian transforms as a scalar with transformations given 
by $U_{k}^{\mathrm{BdG}}(g) H_{k}^{\mathrm{BdG}} U_{\boldsymbol{k}}^{\mathrm{BdG}}(g)^{\dagger}=H_{g k}^{\mathrm{BdG}}$, where

$$
U_{k}^{\mathrm{BdG}}(g)=\left(\begin{array}{cc}
U_{\boldsymbol{k}}(g) & 0 \\
0 & \chi_{g} U_{-k}^{*}(g)
\end{array}\right) .
$$

Note that $\chi_{g} \in U(1)$ is the eigenvalue of the order parameter under $g, U_{k}(g) \Delta_{k} U_{-k}^{T}(g)=\chi_{g} \Delta_{g k}$, in other words the character of the irreducible representation to which $\Delta_{k}$ belongs. ${ }^{4}$ Finally, $H_{k}^{\mathrm{BdG}}$ obeys PHS $\mathcal{P}$, with $\mathcal{P}=\tau_{x} K$ acting in Nambu space and $\tau_{i}$ the Pauli matrices.

At TRIMs, each eigenstate of $H_{k}^{\mathrm{BdG}}$ belongs to either the even or odd irreducible representation of inversion $\mathcal{I}$, corresponding to an inversion eigenvalue $\alpha_{k}= \pm 1$. Furthermore, $\Delta_{k}$ is either even or odd under inversion, meaning $\chi_{\mathcal{I}}= \pm 1$.

For the aforementioned construction, we need $n_{k \text {,BdG }}^{\alpha}$, the number of occupied BdG bands transforming as the irreducible representation $\alpha$ at TRIM $\boldsymbol{k}$. The $n_{\boldsymbol{k}, \mathrm{BdG}}^{\alpha}$ can be deduced from the normal-state transformation properties by assuming weak coupling. In this limit, the transformation properties of the BdG bands at the TRIMs do not change for $\Delta_{k} \rightarrow 0 .{ }^{5}$ Then, the transformation behavior of the BdG bands is entirely specified by the normal-state properties and $\chi_{\mathcal{I}}$. It follows from Eq. (5) that the irreducible representations of eigenstates of $H_{k}^{*}$ are given by $\chi_{\mathcal{I}} \alpha_{-k}$ [27]. Each eigenstate of the normal state Hamiltonian at a TRIM with energy $E_{k}$ and eigenvalue $\alpha_{k}$ is thus mapped to two eigenstates in the BdG Hamiltonian, namely $\left(E_{k}, \alpha_{k}\right)$ and $\left(-E_{k}, \chi_{\mathcal{I}} \alpha_{k}\right)$.

For the difference in the number of irreducible representations in the BdG bands between $\mu=\epsilon_{\mathrm{F}}$ and $\mu=-\infty$ as defined in Eq. (3), only occupied bands of the original normal state are relevant. In particular, Eq. (3) quantifies the difference of the occupied bands stemming from the normal (electron) states and the down-folded (hole) states. It follows from $\alpha_{\mathbf{k}}=\chi_{\mathcal{I}} \alpha_{-\mathbf{k}}$ for the latter that $\kappa_{d, \xi} \equiv 0$ for inversioneven order parameters, $\chi_{\mathcal{I}}=1$, because $\kappa_{d, \xi}$ becomes independent of $\mu$. For inversion-odd order parameters, we find Eq. (1).

Let us inspect the stability of $\kappa_{d, \xi}$ against adding trivial normal-state AL bands below the Fermi level. Such additions should not change the topology of the system as deduced from the indicator. Specifically, in 1D, two TRIMs contribute to the inversion-eigenvalue count and hence, adding a fully filled trivial band below the chemical potential, in other words a band with $r_{\xi} \sum_{k}\left(n_{k, N}^{+}-n_{k, N}^{-}\right)=2 n(n \in \mathbb{Z})$, will change the index by $2 n$. We conclude that $\kappa_{1, \xi}$ is a $\mathbb{Z}_{2}$ quantity. In general, $r_{\xi} \sum_{k}\left(n_{k, N}^{+}-n_{k, N}^{-}\right)=2^{d} n$ in $d$ dimensions from which it follows that $\kappa_{d, \xi}$ is a $\mathbb{Z}_{2^{d}}$ quantity.

In the following, we discuss the cases illustrated in Table I for one, two, and three dimensions and provide details about their construction.

\footnotetext{
${ }^{4}$ For higher-dimensional irreducible representations, a gap function breaks additional (normal-state) symmetries. In the reduced symmetry group, the gap function belongs to a $1 \mathrm{D}$ irreducible representation.

${ }^{5}$ Note that a weaker assumption is sufficient, namely that the order parameter does not change the ordering of the bands at the highsymmetry points.
}

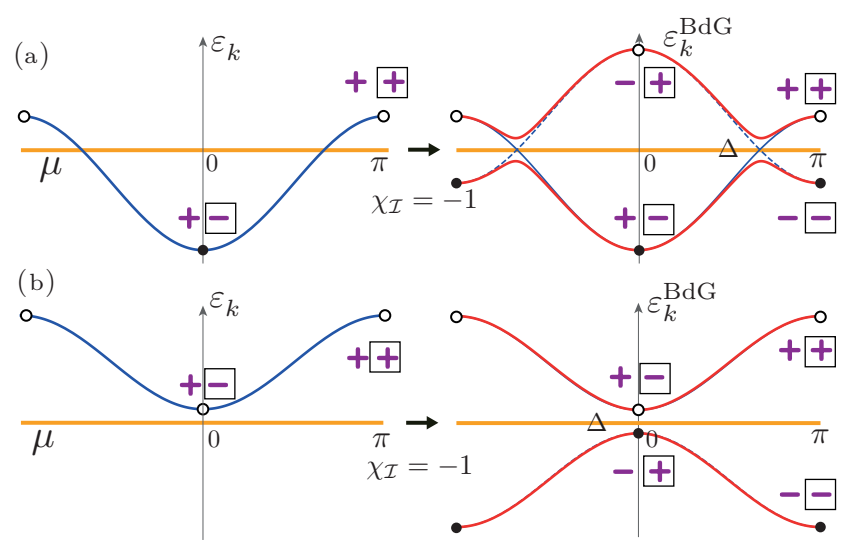

FIG. 1. The BdG spectrum $\varepsilon_{k}^{\text {BdG }}$ obtained from a partially filled (empty) normal band $\varepsilon_{k}$ and an inversion-odd order parameter $\Delta_{k}$. The inversion eigenvalues at TRIMs $k=\{0, \pi\}$ for two different inversion representations $\mathcal{I}=\tau_{z}$ and $\mathcal{I}=-e^{i k} \tau_{z}$ are indicated by unboxed and boxed symbols \pm , respectively.

\section{A. One-dimensional case}

To illustrate the importance of an AL for symmetry indicators of BdG bands, we consider a $1 \mathrm{D}$ chain of spinless fermions. The normal-state Hamiltonian in momentum space is given by a tight-binding nearest-neighbor model with dispersion $\varepsilon_{k}=-2 t \cos (k)-\mu$ with hopping parameter $t$. For spinless fermions, the order parameter is necessarily odd, $\Delta_{k}=-\Delta_{-k}$ or $\chi_{\mathcal{I}}=-1$. The BdG Hamiltonian reads $H_{k}^{\mathrm{BdG}}=\varepsilon_{k} \tau_{z}+\Delta_{k} \tau_{x}$, has inversion $\mathcal{I}$ with $\mathcal{I} H_{k}^{\mathrm{BdG}} \mathcal{I}^{-1}=$ $H_{-k}^{\mathrm{BdG}}$, and belongs to class $\mathrm{D}$. In $1 \mathrm{D}$, class $\mathrm{D}$ is characterized by a $\mathbb{Z}_{2}$ topological invariant. This invariant does not depend on the symmetry properties of the underlying normal-state bands or the presence and nature of fully occupied bands. In particular, the system is topologically nontrivial if an odd number of Fermi surfaces exists [29].

Figure 1 illustrates the ambiguity of symmetry eigenvalues of normal and BdG band structures for the topologically trivial (empty) and nontrivial (partially filled) situation. There are four possibilities for the representation of inversion symmetry of a single nondegenerate band depending on the symmetry and location of the Wannier orbitals compared to the inversion center. When their locations coincide, the representation of inversion is given by $\mathcal{I}= \pm \tau_{z}$, with \pm reflecting the symmetry of the underlying orbitals, for example $s$ and $p$ orbitals, respectively, and $\tau_{z}$ follows from Eq. (5). If the locations of the orbitals and the inversion center are shifted by half a lattice constant, $\mathcal{I}= \pm \tau_{z} e^{i k}$. For clarity, we only show the normalstate cases $\alpha_{k}=1$ and $\alpha_{k}=-e^{i k}$ in Fig. 1. The resulting BdG eigenvalues are opposite for these two cases for the partially filled (topological) and the empty (trivial) situation. Clearly, focusing solely on the BdG eigenvalues cannot indicate a topological phase in 1D. Calculating the indicator as given in Eq. (1), however, distinguishes the topological phase. Note that the situation in Fig. 1(b) exactly corresponds to the AL as defined at the beginning of this section. Table II summarizes all possible BdG eigenvalues and their connection to the (non) trivial topology.

While we have only discussed the case without TRS above, adding TRS in the presence of inversion simply doubles 
TABLE II. Possible inversion eigenvalues $\alpha_{k}$ at TRIMs $k=\{0, \pi\}$ for a 1D single-band BdG Hamiltonian (class D). Shaded cells indicate occupied normal states. The resulting $v_{1,1, \mathrm{D}}=\kappa_{1, \mathrm{D}} \bmod 2$ correctly identifies the topological phase. Note that $\mu<-2 t$ corresponds to the AL defined in the text.

\begin{tabular}{|c|c|c|c|c|c|c|c|c|}
\hline \multirow[b]{3}{*}{$\mathcal{I}$} & \multicolumn{8}{|c|}{$\alpha_{k}$} \\
\hline & \multicolumn{2}{|c|}{$\mu<-2|t|$} & \multicolumn{4}{|c|}{$-2|t|<\mu<2|t|$} & \multicolumn{2}{|c|}{$\mu>2|t|$} \\
\hline & 0 & $\pi$ & 0 & $\pi$ & 0 & $\pi$ & 0 & $\pi$ \\
\hline$\tau_{z}$ & - & - & + & - & - & + & + & + \\
\hline$-\tau_{z}$ & + & + & - & + & + & - & - & - \\
\hline$\tau_{z} e^{i k}$ & - & + & + & + & - & - & + & - \\
\hline$-\tau_{z} e^{i k}$ & + & - & - & - & + & + & - & + \\
\hline $\mathcal{v}_{1,1, \mathrm{D}}$ & & & & & & & & \\
\hline
\end{tabular}

the number of bands. Since both cases in $1 \mathrm{D}$ have a $\mathbb{Z}_{2}$ topological invariant, $\kappa_{1, \xi}$ fully captures the topological nature of the phase for inversion-symmetric systems.

\section{B. Two-dimensional case}

In 2D without TRS, class $\mathrm{D}$, the strong topological phase hosts chiral Majorana edge states and is characterized by a Chern number $C \in \mathbb{Z}$. Thus the indicator only identifies first-order topological phases with odd $C, C \bmod 2=v_{2,1, \mathrm{D}}$. In class DIII, helical Majorana edge states exist in the topological phase and the $\mathbb{Z}_{2}$ invariant characterizing this first-order topological phase [6] coincides with the indicator $v_{2,1, \text { DIII }}$ for systems with inversion symmetry.

When $v_{2,1, \mathrm{D}}=0$, the system may still be in a strong topological phase with even but nonzero Chern number. Assuming $C=0, v_{2,2, \mathrm{D}}=1$ indicates a second-order TSC with two corner Majorana states in an inversion-symmetric geometry (see Table I, $n=2, d=2$ ). This higher-order phase is thus the "minimal" topology required by $v_{2,2, \mathrm{D}}=1$. One can obtain such a superconductor from a strong TSC with $\nu_{2,1, \mathrm{DIII}}=1$ by introducing a TRS-breaking mass term that preserves $\mathcal{I}$ [11].

A TRS second-order TSC is indicated by $v_{2,2, \text { DIII }}=1$ and features Kramer's paired Majorana corner modes. It can be obtained by adding the fully occupied band of a $\mathbb{Z}_{2}$ TI in symmetry class AII to a trivial odd-parity superconductor. ${ }^{6}$

\section{Three-dimensional case}

The strong TSCs in 3D with TRS and PHS belong to class DIII and are characterized by the winding number $W \in \mathbb{Z}$ [6]. As with $C$ in 2D, $\kappa_{3 \text {,DIII }}$ identifies first-order topological

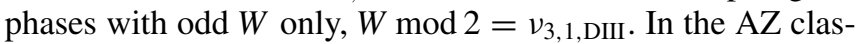
sification, class D has no gapped topological phase, but with translational symmetry $v_{3,1, \mathrm{D}}=1$ indicates momentum-space planes with different $C$ and thus gapless Weyl superconductors [32,33].

When $v_{3,1, \mathrm{DIII}}=0$, the system may still be in a strong topological phase with even but nonzero winding number $W$. Assuming $W=0, v_{3,2 \text {,DIII }}=1$ indicates a second-order TSC with a Kramer's pair of helical hinge Majoranas in

\footnotetext{
${ }^{6}$ See Ref. [18] for a related proposal in symmetry class AIII.
}

an inversion-symmetric geometry. Further, $v_{3,2, \mathrm{DIII}}=0$ but $v_{3,3 \text {, DIII }}=1$ indicates a third-order TSC with a Kramer's pair of Majorana corner modes. These higher-order phases have again the minimal topology compatible with the respective values of the symmetry indicators. The second- and thirdorder phases can be obtained by adding the fully occupied band of, respectively, a $3 \mathrm{D} \mathbb{Z}_{2}$ TI in symmetry class AII and a higher-order TI with $\kappa_{1} \bmod 4=2$ to an otherwise trivial odd-parity superconductor.

We close by constructing examples of the bulk-boundary correspondence of all higher-order phases in class D and DIII shown in Table I using surface Dirac theories. We follow Ref. [11] and start from a (first-order) strong topological phase in DIII with winding number $W= \pm 1$ and described by the bulk Hamiltonian $\mathcal{H}_{ \pm}$for an odd-parity superconductor with a single Fermi pocket enclosing the $\Gamma$ point in the normal state. This Hamiltonian is gapped in the bulk but has a Majorana surface cone governed by

$$
h_{ \pm}=\mp\left(\boldsymbol{k} \times \boldsymbol{n}_{\boldsymbol{r}}\right) \cdot \boldsymbol{\tau},
$$

where $\boldsymbol{\tau}$ are Pauli matrices in particle-hole space and $\boldsymbol{n}_{\boldsymbol{r}}$ is the surface normal vector. The surface theory possesses PHS, TRS, and inversion, which on the surface are given by $\mathcal{T}=$ $\tau_{y} \mathcal{K}, \mathcal{P}_{\boldsymbol{r}}=\mp \boldsymbol{n}_{\boldsymbol{r}} \cdot \boldsymbol{\tau} \tau_{y} \mathcal{K}$, and $\mathcal{I}=\mp \tau_{0}$. Given a bulk normalstate inversion eigenvalue of the occupied band at $\Gamma$, the sign of $W$ determines the sign of $\mathcal{I}$ on the surface [11].

We can construct all higher-order phases by adding copies with opposite winding numbers (class DIII) and breaking TRS (class D). A second-order TSC in class D is obtained by adding to the surface Hamiltonian Eq. (6) the only $\boldsymbol{k}$ independent TRS-breaking term that preserves PHS, $\tilde{M}_{\boldsymbol{r}} \boldsymbol{n}_{\boldsymbol{r}} \cdot \boldsymbol{\tau}$. Inversion symmetry requires $\tilde{M}_{r}=-\tilde{M}_{-r}$, which implies two domains with opposite mass sign of this surface Dirac theory. Along the domain wall, which is an inversion symmetric path on the surface, a chiral mode remains (see Fig. 2). ${ }^{7}$

The class DIII second-order phase is built by taking two copies with opposite winding numbers, $\mathcal{H}=\mathcal{H}_{+} \oplus \mathcal{H}_{-}$, leading to $W_{\text {tot }}=0$. The surface theory reads $h_{2}=-\left(\boldsymbol{k} \times \boldsymbol{n}_{\boldsymbol{r}}\right)$. $\boldsymbol{\tau} \gamma_{z}$, with Pauli matrices $\boldsymbol{\gamma}$ acting on the $+/-$ grading. We can

\footnotetext{
${ }^{7}$ Note that the same Hamiltonian at $k_{z}=0$ describes a 2D secondorder TSC in class D.
} 

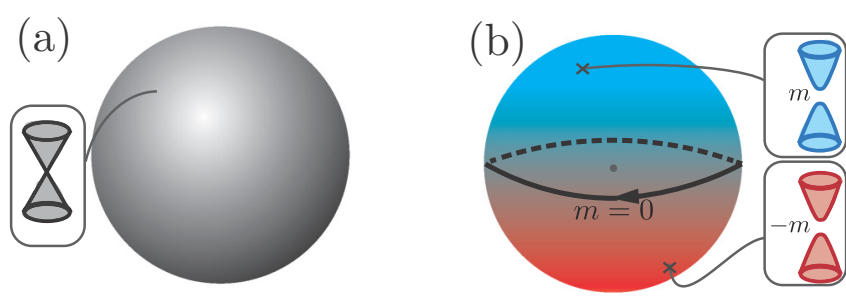

FIG. 2. Construction of a 3D second-order TSC in class D from a first-order TSC in class DIII with a Majorana surface cone (a). Adding a TRS-breaking mass term respecting inversion symmetry, $\tilde{M}_{r}=-\tilde{M}_{-r}$, gaps out the surface except for an inversion-symmetric path, where a chiral Majorana mode remains (b).

add a mass term $M_{\boldsymbol{r}} \gamma_{x}$, which preserves all the symmetries of the Hamiltonian, iff $M_{r}=-M_{-r}$. For the surface theory, these symmetries are represented by $\mathcal{T}=\tau_{y} \mathcal{K}, \mathcal{P}_{\boldsymbol{r}}=-\gamma_{z} \boldsymbol{n}_{\boldsymbol{r}} \cdot \boldsymbol{\tau} \tau_{y} \mathcal{K}$, and $\mathcal{I}=-\gamma_{z}$. The form of $\mathcal{I}$ follows from the requirements $W_{\text {tot }}=0$ and $v_{3,2 \text {, DIII }}=1$. $M_{\boldsymbol{r}}$ being an odd function on the surface enforces again two domains with opposite mass sign. A gapless Kramer's pair of helical Majorana modes is bound to the domain wall, which is an inversion symmetric path on the surface.

The third-order TSC in class D can be obtained from this second-order TSC in class DIII by adding a TRS breaking mass term $\tilde{M}_{r} \gamma_{y}$ with $\tilde{M}_{r}=-\tilde{M}_{-r}$ to the Hamiltonian, corresponding to applying a magnetic field, for instance. This gaps the helical modes except for two inversion-related points on the surface, where two corner Majorana modes remain.

Finally to build a third-order TSC with TRS we combine four copies of $\mathcal{H}_{ \pm}$with $W_{\text {tot }}=0$, i.e., $\mathcal{H}_{+} \oplus \mathcal{H}_{-} \oplus \mathcal{H}_{+} \oplus \mathcal{H}_{-}$. The surface theory reads $h_{4}=-\left(\boldsymbol{k} \times \boldsymbol{n}_{\boldsymbol{r}}\right) \cdot \boldsymbol{\tau} \gamma_{z} \sigma_{0}$, where the Pauli matrices $\sigma$ act on the additional grading. Here, $\mathcal{T}=$ $\tau_{y} \sigma_{0} \mathcal{K}, \mathcal{P}_{\boldsymbol{r}}=-\gamma_{z} \boldsymbol{n}_{\boldsymbol{r}} \cdot \boldsymbol{\tau} \tau_{y} \sigma_{0} \mathcal{K}$, and $\mathcal{I}=-\gamma_{z} \sigma_{0}$. The form of $\mathcal{I}$ follows from the requirements $W_{\text {tot }}=0, v_{3,2 \text {,DIII }}=0$, and $v_{3,3 \text {, DIII }}=1$. Each of the four constituent strong TSC of class DIII features a Majorana surface cone. Coupling these cones pairwise by adding the mass term $M_{r} \gamma_{x} \tau_{0} \sigma_{0}$, with $M_{\boldsymbol{r}}=$ $-M_{-r}$, gaps out the surface states, but leaves two inversionsymmetric gapless lines carrying a helical Majorana mode each. Then, another mass term $\tilde{M}_{r} \gamma_{y} \sigma_{y}$ with $\tilde{M}_{r}=-\tilde{M}_{-r}$ can be added, which anticommutes with the Hamiltonian and preserves all its symmetries, to gap the Majorana edge modes but leave Majorana corner modes at inversion-symmetric points.

\section{CONCLUSIONS}

We presented a unified symmetry indicator for spin-orbit coupled TSCs with inversion symmetry and odd-parity order parameter. The indicator can be evaluated using only the normal state band structure and describes topological superconductors according to the AZ classification as well as higher-order TSCs with hinge and corner Majorana modes. Our analysis is based on the definition of a physical trivial limit for superconductors and can be readily generalized to other spatial symmetries besides inversion (see Appendix). The indicator, Eq. (1), can be used straight-forwardly for model building or tuning and designing new topological su- perconductors, e.g., via doping a topologically trivial oddparity superconductor.

While a brute-force search for topological superconductors, analogous to that of Refs. [24-26], is not possible and, short of a complete $a b$ initio theory of unconventional superconductivity, will not be, our indicator can help in identifying topologically nontrivial superconductors. Once a superconductor is established as being odd-parity, our indicator allows for a straightforward evaluation given only the normal state band structure to identify or exclude the candidate material. An intriguing 2D material, where our indicator could be applied, is the quantum spin Hall insulator monolayer $\mathrm{WTe}_{2}$, which becomes superconducting upon gating. Recent work proposed this state to be odd parity and, in agreement with our indicator, a higher-order TSC [18]. Potential 3D odd-parity superconductors include ferromagnetic uranium compounds, such as $\mathrm{UGe}_{2}$, UCoGe, and URhGe [34], or the nonmagnetic end member of this series, $\mathrm{UTe}_{2}$ [35]. Further study of the normal state band structure is, however, necessary in all these cases to apply our indicator.

Finally, from a more academic point of view, our main insight, namely the need for a trivial "atomic" limit results in a compact, unified topological invariant for superconductors across different dimensions and orders of topology.

Note added. Recently, we became aware of Ref. [36], which primarily discusses symmetry indicators for TSCs in symmetry class BDI and then generalizes to symmetry class D. Our results agree where they overlap. Reference [37] introduces a different but equivalent atomic limit for $\mathrm{BdG}$ Hamiltonians.

\section{ACKNOWLEDGMENTS}

We thank Piet Brouwer, Luka Trifunovic, Ronny Thomale, and Frank Schindler for stimulating discussions. This project has received funding from the European Research Council (ERC) under the European Union's Horizon 2020 research and innovation programm (ERC-StG-Neupert-757867PARATOP).

\section{APPENDIX: SYMMETRY INDICATOR FOR GENERAL SYMMETRY ELEMENT}

In the main text, we have discussed how to obtain the symmetry indicator for superconductors with inversion symmetry. Here, we briefly sketch how symmetry indicators for other point group elements can be obtained following largely the notation of Ono et al., Ref. [27]. We start with the normalstate Hamiltonian $H_{k}$, which transforms under an element of the generating point group, $g \in \mathcal{G}$, as $U_{k}(g) H_{k} U_{k}(g)^{\dagger}=H_{g k}$. The normal-state Hamiltonian enters the BdG Hamiltonian describing the superconducting phase together with the order parameter $\Delta_{k}$ through

$$
H_{k}^{\mathrm{BdG}}=\left(\begin{array}{cc}
H_{k} & \Delta_{k} \\
\Delta_{k}^{\dagger} & -H_{-k}^{*}
\end{array}\right) .
$$

The BdG Hamiltonian transforms under the action of an element $g \in \mathcal{G}$ as $U_{\boldsymbol{k}}^{\mathrm{BdG}}(g) H_{\boldsymbol{k}}^{\mathrm{BdG}} U_{\boldsymbol{k}}^{\mathrm{BdG}}(g)^{\dagger}=H_{g \boldsymbol{k}}^{\mathrm{BdG}}$, 
where

$$
U_{\boldsymbol{k}}^{\mathrm{BdG}}(g)=\left(\begin{array}{cc}
U_{\boldsymbol{k}}(g) & 0 \\
0 & \chi_{g} U_{-k}^{*}(g)
\end{array}\right) .
$$

Finally, the order parameter transforms under $g$ according to $U_{k}(g) \Delta_{k} U_{-k}^{T}(g)=\chi_{g} \Delta_{g k}$, with $\chi_{g} \in U(1)$ the character of the irreducible representation of $g$ to which $\Delta_{k}$ belongs.

Within a weak-coupling approximation, the eigenstates of $H_{k}^{\mathrm{BdG}}$ are directly related to the eigenstates of $H_{k}$ and the irreducible representations of the $\mathrm{BdG}$ bands can be expressed in terms of the ones of the normal metal bands and $\Delta_{k}$. Denoting an irreducible representation of $g$ in the little group $G_{k}$ of $\boldsymbol{k}$ for a band of $H_{k}$ with $u_{k}^{\alpha}(g)$ with $\alpha$ the eigenvalue of $g$ labeling the irreducible representation, the irreducible representation of the same band of $H_{-k}^{*}$ is given by $\chi_{g} u_{-k}^{\alpha}(g)^{*}$. We thus define a map $\alpha \mapsto f_{k}(\alpha)$ from the irreducible representation of the "electron" band from $H_{k}$ to the "hole" band given by $H_{-k}^{*}$ through $\chi_{g} u_{-k}^{\alpha}(g)^{*}=u_{k}^{f_{k}(\alpha)}(g)$ [27]. Note that with $g=\mathcal{I}$ and thus $\alpha= \pm 1$, the relation simplifies to $f(\alpha)=\chi_{\mathcal{I}} \alpha$.
We can now define a symmetry indicator for any element of the generating point group, $g \in \mathcal{G}$, as

$$
\begin{aligned}
\kappa_{d, \xi}^{g} & =r_{d, \xi}^{g} \sum_{k \in K_{g}} \sum_{\alpha} \frac{\alpha}{2}\left(\left.n_{k, \mathrm{BdG}}^{\alpha}\right|_{\mu=\epsilon_{F}}-\left.n_{\boldsymbol{k}, \mathrm{BdG}}^{\alpha}\right|_{\mu=-\infty}\right) \\
& =r_{d, \xi}^{g} \sum_{\boldsymbol{k} \in K_{g}} \sum_{\alpha} \frac{\alpha}{2}\left(n_{\boldsymbol{k}, \mathrm{N}}^{\alpha}-n_{\boldsymbol{k}, \mathrm{N}}^{f_{k}(\alpha)}\right),
\end{aligned}
$$

where $n_{k, N}^{\alpha}$ is the number of occupied energy bands of the normal state Hamiltonian belonging to the irreducible representation $u_{\boldsymbol{k}}^{\alpha}(g)$ at momentum $\boldsymbol{k}$ and the sum is over momenta $\boldsymbol{k} \in K_{g}$ whose little group contains $g$. The prefactor $r_{d, \xi}^{g}$ depends on the symmetry element $g$, space dimension $d$, and the AZ symmetry class $\xi=\{D, D I I I\}$. Note that this formalism has been used in Ref. [38] for a classification using all 230 space groups. In case of inversion symmetry there are only two inversion eigenvalues $\alpha \in\{+1,-1\}$ and the formula for symmetry-indicator simplifies to the one given in the main text, Eq. (1).
[1] A. Y. Kitaev, Phys. Usp. 44, 131 (2001).

[2] N. Read and D. Green, Phys. Rev. B 61, 10267 (2000).

[3] D. A. Ivanov, Phys. Rev. Lett. 86, 268 (2001).

[4] M. Sato and Y. Ando, Rep. Prog. Phys. 80, 076501 (2017).

[5] A. P. Schnyder, S. Ryu, A. Furusaki, and A. W. W. Ludwig, Phys. Rev. B 78, 195125 (2008).

[6] S. Ryu, A. P. Schnyder, A. Furusaki, and A. W. W. Ludwig, New J. Phys. 12, 065010 (2010).

[7] W. A. Benalcazar, B. A. Bernevig, and T. L. Hughes, Science 357, 61 (2017).

[8] F. Schindler, A. M. Cook, M. G. Vergniory, Z. Wang, S. S. P. Parkin, B. A. Bernevig, and T. Neupert, Sci. Adv. 4, eaat0346 (2018).

[9] M. Geier, L. Trifunovic, M. Hoskam, and P. W. Brouwer, Phys. Rev. B 97, 205135 (2018).

[10] L. Trifunovic and P. W. Brouwer, Phys. Rev. X 9, 011012 (2019).

[11] E. Khalaf, Phys. Rev. B 97, 205136 (2018).

[12] E. Khalaf, H. C. Po, A. Vishwanath, and H. Watanabe, Phys. Rev. X 8, 031070 (2018).

[13] E. Lee, R. Kim, J. Ahn, and B.-J. Yang, arXiv:1904.11452.

[14] X.-L. Sheng, C. Chen, H. Liu, Z. Chen, Y. X. Zhao, Z.-M. Yu, and S. A. Yang, arXiv:1904.09985.

[15] J. Langbehn, Y. Peng, L. Trifunovic, F. von Oppen, and P. W. Brouwer, Phys. Rev. Lett. 119, 246401 (2017).

[16] Y. Wang, M. Lin, and T. L. Hughes, Phys. Rev. B 98, 165144 (2018).

[17] S. Sumita, T. Nomoto, K. Shiozaki, and Y. Yanase, Phys. Rev. B 99, 134513 (2019).

[18] Y.-T. Hsu, W. S. Cole, R.-X. Zhang, and J. D. Sau, arXiv: 1904.06361

[19] J. Kruthoff, J. de Boer, J. van Wezel, C. L. Kane, and R.-J. Slager, Phys. Rev. X 7, 041069 (2017).
[20] H. C. Po, A. Vishwanath, and H. Watanabe, Nat. Commun. 8 , 50 (2017).

[21] B. Bradlyn, L. Elcoro, J. Cano, M. G. Vergniory, Z. Wang, C. Felser, M. I. Aroyo, and A. Bernevig, Nature (London) 547, 298 (2017).

[22] S. Ono and H. Watanabe, Phys. Rev. B 98, 115150 (2018).

[23] L. Fu, C. L. Kane, and E. J. Mele, Phys. Rev. Lett. 98, 106803 (2007).

[24] F. Tang, H. C. Po, A. Vishwanath, and X. Wan, Nat. Phys. 15, 470 (2019).

[25] T. Zhang, Y. Jiang, Z. Song, H. Huang, Y. He, Z. Fang, H. Weng, and C. Fang, Nature 566, 475 (2019).

[26] M. G. Vergniory, L. Elcoro, C. Felser, N. Regnault, B. A. Bernevig, and Z. Wang, Nature 566, 480 (2019).

[27] S. Ono, Y. Yanase, and H. Watanabe, Phys. Rev. Res. 1, 013012 (2019).

[28] M. Sato, Phys. Rev. B 79, 214526 (2009).

[29] M. Sato, Phys. Rev. B 81, 220504(R) (2010).

[30] L. Fu and E. Berg, Phys. Rev. Lett. 105, 097001 (2010).

[31] X.-L. Qi, T. L. Hughes, and S.-C. Zhang, Phys. Rev. B 81, 134508 (2010).

[32] T. Meng and L. Balents, Phys. Rev. B 86, 054504 (2012).

[33] M. H. Fischer, T. Neupert, C. Platt, A. P. Schnyder, W. Hanke, J. Goryo, R. Thomale, and M. Sigrist, Phys. Rev. B 89, 020509(R) (2014).

[34] V. P. Mineev, Phys. Usp. 60, 121 (2017)

[35] S. Ran, C. Eckberg, Q.-P. Ding, Y. Furukawa, T. Metz, S. R. Saha, I.-L. Liu, M. Zic, H. Kim, J. Paglione, and N. P. Butch, Science 365, 684 (2019).

[36] J. Ahn and B.-J. Yang, arXiv:1906.02709.

[37] M. Geier, P. W. Brouwer, and L. Trifunovic, arXiv:1910.11271.

[38] S. Ono, H. C. Po, and H. Watanabe, arXiv:1909.09634. 